\title{
Situación nutricional del escolar y adolescente en Chile
}

\author{
LORENA RODRÍGUEZ O. ${ }^{1}$
}

\section{Introducción}

El perfil epidemiológico de las enfermedades del chileno ha cambiado notoriamente en las últimas décadas. Las diferentes acciones en salud, la generación de un mayor conocimiento científico nacional en áreas prioritarias y la mejoría en las condiciones de saneamiento ambiental entre otras, han permitido erradicar la desnutrición infantil y disminuir la prevalencia de las enfermedades infecciosas. Esto se ha traducido en una disminución de la mortalidad infantil de 125 por 1000 nacidos vivos en la década de los 60 a 16 por 1000 a comienzos de 1990, llegando actualmente a cifras de 7,8 por 1000.

Los datos de vigilancia epidemiológica que disponemos, a través de las estadísticas del Ministerio de Salud, demuestran que la población infantil, de menores de 6 años, presenta cifras cada vez menores de bajo peso y un incremento importante en los niveles de malnutrición por exceso. Esta realidad no es ajena a lo que sucede en otros países en desarrollo o desarrollados, en los que al igual que en nuestro país, los cambios desfavorables en los estilos de vida están determinando un perfil epidemiológico caracterizado por el sedentarismo, los malos hábitos alimentarios y las enfermedades crónicas no transmisibles (ECNTs). Estas patologías, que representan una impor- tante carga en morbilidad y mortalidad en la edad adulta, se originan en gran parte, muy precozmente en la etapa infantil, incluso antes, en la etapa fetal, y se incrementan durante toda la vida.

Recientemente el Ministerio de Salud inició para todo el país una "Estrategia de Intervención Nutricional a través del Ciclo Vital", que pretende relevar el tema nutricional, a través de la consejería en vida sana, en todas las acciones de salud, con énfasis en la infancia y en el embarazo, con el objetivo de prevenir las ECNTs.

Actualmente, existen diversas iniciativas, tanto en el ámbito de salud como de educación, y en conjunto con grupos académicos expertos, cuyo objetivo es contribuir a la promoción de la salud y a la prevención tanto de la malnutrición por exceso como de las enfermedades asociadas a ella (diabetes, hipertensión, cardiovasculares y otras).

El Ministerio de Salud en respuesta a la necesidad de concordar la forma de evaluar a los escolares y adolescentes formuló en el año 2004, la norma técnica de evaluación del estado nutricional del niño(a) de 6 a 18 años, lo que constituye el primer paso para avanzar en la tarea de intervenir también en este grupo etario.

No podemos dejar de lado otro grupo de enfermedades del ámbito nutricional, que afectan a los escolares, y especialmente a los adolescentes, y que tiene que ver con alteraciones

\section{Departamento de Nutrición y Ciclo Vital. Ministerio de Salud Chile.}

Correspondencia a:

Dra. Lorena Rodríguez O.

E-mail: Irodriguez@minsal.cl 
en la conducta alimentaria, de distintos niveles de gravedad, y que están relacionadas con un cambio en el modelo de belleza y éxito y que también debemos enfrentar desde la mirada de la promoción de la salud y de la prevención en grupos de riesgo.

\section{Programa de Salud de las y los Adolescentes del Ministerio de Salud}

Este programa, de carácter nacional, esta destinado a poner al alcance de las y los adolescentes un conjunto de intervenciones con vocación de derechos ciudadanos en salud pública, destinadas a actuar sobre los factores de riesgo o determinantes de la salud actual y futura de mayor significación en esta etapa de la vida.

Actualmente, de las todas las atenciones entregadas a la población por el sistema público de salud, alrededor del $10 \%$ tiene una o un adolescente como beneficiario, tanto en atención primaria y especialidades como en urgencia. Además, alrededor del $23 \%$ de las mujeres en control de embarazo y el $11 \%$ de las mujeres en planificación familiar son menores de 19 años. Sin embargo, las atenciones que están dirigidas a la prevención y detección precoz de riesgos y enfermedades son claramente insuficientes.

Este año 2005, con el propósito de facilitar y estimular el acceso de la población adolescente a las atenciones, principalmente preventivas y promocionales y en el Marco de la Instalación de la nuevas leyes que orientan la reforma, el Plan de Salud Pública Nacional ha establecido, como una de sus acciones, la instalación de una modalidad de atención amigable para adolescentes y jóvenes en los establecimientos de atención primaria. Esta reorientación de los servicios dirigidos las personas de 10 a 19 años ha sido diseñada a partir de la evidencia científica y de la experiencia chilena e internacional de las últimas décadas.

Las prioridades para el 2005 son: reducir el habito de fumar, reducir la obesidad, reducir el sedentarismo, reducir los embarazos no deseados, reducir la tasa de suicidio, reducir la mortalidad por traumatismos y accidentes de tránsito, disminuir la recurrencia de episodios depresivos, reducir la tasa de beber problema y de consumo de drogas y disminuir las caries dentales.

\section{Norma para la Evaluación Nutricional del Escolar y Adolescente}

Tradicionalmente la evaluación nutricional de los niños en Chile se realizaba con el indicador peso para la talla, sin embargo, la evidencia científica demuestra que el índice de masa corporal (IMC) presenta mejor correlación que el $\mathrm{P} / \mathrm{T}$ con la composición corporal. Considerando estos antecedentes, el Ministerio de Salud en conjunto con un grupo de académicos expertos en el tema desarrolló en el año 2004, la Norma Técnica de evaluación nutricional para este grupo etario, la cual considera edad, sexo y grado de maduración sexual, lo que permite establecer diferencias individuales para el diagnóstico del estado nutricional. Los estándares de comparación son los americanos del National Center for Chronic Disease-National Center for Health Statistics (CDC-NCHS).

El IMC es el indicador recomendado por la OMS para evaluar antropométricamente el estado nutricional de una población menor de 20 años, por su simpleza, bajo costo y adecuada correlación con la grasa corporal total. Sin embargo, esta correlación varía con la madurez biológica y disminuye de 0,90 en los prepúberes a 0,32 en los púberes. Por ello, diferentes autores señalan la importancia de ser cautelosos en el diagnóstico de la obesidad durante la pubertad al utilizar este indicador, y la necesidad de mejorar su sensibilidad considerando la raza, el sexo y el grado de desarrollo puberal alcanzado.

En un estudio de Burrows, Díaz y Muzzo, en una muestra elegida de escolares chilenos se observó un rango de 6 años en la edad de inicio de la pubertad, encontrando mujeres en M2 entre los 8 y los 14 años y varones en G2 entre los 10 y los 16 años. Por otra parte, entre los 9 y los 13 años en las mujeres y entre los 12 y 16 años en los varones, se observaron niños en todas las etapas de desarrollo puberal. Estos hallazgos son similares a los observados en población americana y europea y confirman la considerable variabilidad maduracional durante la pubertad entre niños de similar edad cronológica. La similitud del IMC en niños de diferentes edades cronológicas pero en igual estadio de Tanner y, por otro lado, el incremento significativo del IMC cuando se comparan niños de igual edad cronológica pero en dife- 
rentes estadios puberales, confirman que el IMC está asociado a la madurez biológica más que a la edad cronológica en esta etapa de la vida. El aumento del IMC y su distribución percentilar fueron muy regulares al estratificarlo por grado de desarrollo puberal alcanzado, a pesar de la variabilidad en la edad cronológica de los niños que se encuentran en una misma etapa de Tanner, confirmando una vez más la relación entre el IMC y la madurez biológica.

El IMC es considerado el mejor indicador antropométrico para diagnosticar el sobrepeso y la obesidad en niños entre los 2 y los 18 años. Estos referentes incluyen a una agrupación de 9 estudios transversales (66 772 niños de ambos sexos, blancos, negros, hispánicos y asiáticos entre 5 y 17 años, realizados entre los años 1970 y 1994 en diferentes estados de los Estados Unidos de Norteamérica (USA) por la NCHS (NHANES II y NHANES III).

La norma técnica propone como indicador para la evaluación del estado nutricional en escolares y adolescentes en el nivel primario de atención, la relación IMC/edad:

$\mathrm{IMC}=$ peso/talla ${ }^{2}$.

La población de referencia se basa en los estándares de comparación americanos del National Center for Chronic Disease-National Center for Health Statistics (CDC-NCHS).

Estas tablas de referencia dan valores cada 6 meses.

\section{Criterios de calificación:}

IMC $<$ p10 bajo peso.

IMC entre $\mathrm{p} 10 \mathrm{y}<\mathrm{p} 85$ normal.

IMC entre $\mathrm{p} 85 \mathrm{y}<\mathrm{p} 95$ riesgo obesidad.

IMC $\geq$ p95 obesidad.

\section{Señales de alarma:}

ascenso del canal de crec del IMC en dos controles sucesivos.

Aumento del IMC $>1,5$ puntos en 6 meses.

La referencia para talla será también CDC EEUU y el criterio calificación será talla para la edad (T/E):

$\mathrm{T} / \mathrm{E}<\mathrm{p} 5$ talla baja

T/E entre p5 y p95 talla normal

\section{Señales de alarma}

Incremento en talla $<2,5 \mathrm{~cm}$ en 6 meses (incremento insuficiente).
Incremento en talla $>3,5 \mathrm{~cm}$ en 6 meses (descartar pubertad precoz).

Descenso del canal de crecimiento en dos controles sucesivos.

$\mathrm{T} / \mathrm{E}<\mathrm{p} 10$.

A nivel secundario considerar grado desarrollo puberal según tablas de maduración sexual y etapas de Tanner. Si la diferencia entre edad cronológica y edad biológica es menor a 1 año prima la edad cronológica para la evaluación del estado nutricional y si ésta diferencia es mayor de 1 año prima la edad biológica. La edad biológica se determina por desarrollo mamario en niñas y por desarrollo de genitales externos en niños.

\section{Estado Nutricional del Preescolar, Escolar y Adolescente}

Las estadísticas del Ministerio de Salud proporcionan (tablas 1, 2 y 3 ) la evaluación nutricional de la población menor de 6 años de todos los beneficiarios del sistema. El análisis de estos datos nos muestra que así como ha habido una sistemática caída de las cifras de desnutrición en los últimos 15 años, ha habido también un aumento alarmante de las cifras de sobrepeso y obesidad. También nos revelan una estabilización del aumento de la malnutrición por exceso en los últimos 2 a 3 años, debido probablemente a numerosas actividades de promoción de salud y de prevención, establecidos en distintos ámbitos de salud y educación, esto es por ejemplo apoyo de la lactancia materna, escuelas saludables, aumento de la difusión de los temas relativos a estilos de vida saludable, campañas promocionales, etc.

Por otro lado, la encuesta nacional de salud (ENS) 2003 reveló cifras alarmantes de obesidad, hipertensión arterial, dislipidemias y sedentarismo en la población mayor de 17 años (tabla 4).

Chile no cuenta con datos nacionales de vigilancia nutricional en la población de escolares y adolescentes. Los datos disponibles emanan de algunos estudios epidemiológicos y de los datos que entrega la Junta Nacional de Auxilio Escolar y Becas (JUNAEB) provenientes de la evaluación nutricional que realizan en primer año básico de escuelas municipalizadas 
RODRÍGUEZ L.

Tabla 1. Situación nutricional de niños y niñas menores de 2 años. Según calificación nutricional

\begin{tabular}{ccccc}
\hline Años & $\begin{array}{c}\text { Riesgo de desnutrir } \\
\text { \% }\end{array}$ & $\begin{array}{c}\text { Desnutrición } \\
\text { \% }\end{array}$ & $\begin{array}{c}\text { Sobrepeso }^{\mathbf{3}} \\
\%\end{array}$ & $\begin{array}{c}\text { Obesidad }^{\mathbf{4}} \\
\mathbf{\%}^{\mathbf{\%}}\end{array}$ \\
\hline 1994 & 6,1 & 1,4 & 17,4 & 5,7 \\
1995 & 5,6 & 1,2 & 16,8 & 5,8 \\
1996 & 5,5 & 1,1 & 16,6 & 5,9 \\
1997 & 5,4 & 1,0 & 16,7 & 6,0 \\
1998 & 1,1 & 16,1 & 5,7 \\
1999 & 5,5 & 1,0 & 16,1 & 5,8 \\
2000 & 5,4 & 0,9 & 16,4 & 5,9 \\
2001 & 5,1 & 0,9 & 16,4 & 6,0 \\
2003 & 5,3 & 0,9 & 16,8 & 6,0 \\
\hline
\end{tabular}

${ }^{1}$ Peso para la edad -1 a $-1,9 \mathrm{DE}$. ${ }^{2}$ Peso para la edad $<-2 \mathrm{DE}$. ${ }^{3}$ Peso para la talla 1 a 1,9 DE. ${ }^{4}$ Peso para la talla $\geq 2 \mathrm{DE}$

Tabla 2. Situación nutricional de niños y niñas entre 2 y 5 años. Según calificación nutricional

\begin{tabular}{ccccc}
\hline Año & $\begin{array}{c}\text { Riesgo de desnutrir } \\
\text { \% }\end{array}$ & $\begin{array}{c}\text { Desnutridos } \\
\text { \% }\end{array}$ & $\begin{array}{c}\text { Sobrepeso }^{\mathbf{3}} \\
\%\end{array}$ & $\begin{array}{c}\text { Obesidad }^{\mathbf{4}} \\
\mathbf{\%}^{\mathbf{0}}\end{array}$ \\
\hline 1994 & 2,0 & 0,4 & 14,9 & 5,9 \\
1995 & 1,9 & 0,4 & 14,5 & 6,2 \\
1996 & 1,7 & 0,4 & 14,9 & 6,4 \\
1997 & 1,7 & 0,4 & 15,2 & 7,1 \\
1998 & 1,8 & 0,4 & 14,8 & 7,3 \\
1999 & 1,9 & 0,4 & 15,0 & 7,7 \\
2000 & 1,9 & 0,4 & 15,0 & 7,8 \\
2001 & 2,1 & 0,3 & 15,4 & 8,1 \\
2002 & 2,1 & 0,3 & 15,5 & 8,1 \\
\hline
\end{tabular}

${ }^{1}$ Peso para la edad $-1 \mathrm{a}-1,9 \mathrm{DE} .{ }^{2}$ Peso para la edad $<-2 \mathrm{DE} .{ }^{3}$ Peso para la talla 1 a 1,9 DE. ${ }^{4}$ Peso para la talla $\geq 2 \mathrm{DE}$

Tabla 3. Diagnóstico nutricional integrado de la población menor de 6 años, según grupo de edad, Ministerio de Salud, Chile 2004

\begin{tabular}{|c|c|c|c|c|c|c|c|c|c|c|c|c|c|}
\hline \multirow[t]{2}{*}{$\begin{array}{l}\text { Grupo de } \\
\text { Salud }\end{array}$} & \multirow[t]{2}{*}{$\begin{array}{l}\text { Población } \\
\text { en control }\end{array}$} & \multicolumn{2}{|c|}{ En riesgo } & \multicolumn{2}{|c|}{ Desnutridos } & \multicolumn{2}{|c|}{ s Sobrepeso } & \multicolumn{2}{|c|}{ Obesos } & \multicolumn{2}{|c|}{ Normal } & \multicolumn{2}{|c|}{$\begin{array}{l}\text { Desnutr. } \\
\text { Secundaria }\end{array}$} \\
\hline & & $\mathbf{n}$ & Tasa & $\mathbf{n}$ & Tasa & $\mathbf{n}$ & Tasa & $\mathbf{n}$ & Tasa & $\mathbf{n}$ & Tasa & $n$ & Tasa \\
\hline Chile & 1008429 & 32524 & 3,2 & 5282 & 0,5 & 158334 & 15,7 & 74962 & 7,4 & 736447 & 73,0 & 880 & 0,1 \\
\hline 0 - 2 meses & 36000 & 211 & 0,6 & 27 & 0,1 & 3843 & 10,7 & 853 & 2,4 & 31057 & 86,3 & 9 & 0,0 \\
\hline 3 - 5 meses & 40837 & 353 & 0,9 & 43 & 0,1 & 7706 & 18,9 & 2511 & 6,1 & 30214 & 74,0 & 10 & 0,0 \\
\hline 6 - 11 meses & 81624 & 3478 & 4,3 & 526 & 0,6 & 15077 & 18,5 & 5630 & 6,9 & 56853 & 69,7 & 60 & 0,1 \\
\hline 12 - 17 meses & 86835 & 6800 & 7,8 & 1172 & 1,3 & 13729 & 15,8 & 5162 & 5,9 & 59855 & 68,9 & 117 & 0,1 \\
\hline $18-23$ meses & 85076 & 6440 & 7,6 & 1083 & 1,3 & 12893 & 15,2 & 5310 & 6,2 & 59247 & 69,6 & 103 & 0,1 \\
\hline $24-47$ meses & 344288 & 10074 & 2,9 & 1617 & 0,5 & 48170 & 14,0 & 19395 & 5,6 & 264721 & 76,9 & 311 & 0,1 \\
\hline 48 - 71 meses & 333769 & 5168 & 1,5 & 814 & 0,2 & 56916 & 17,1 & 36101 & 10,8 & 234500 & 70,3 & 270 & 0,1 \\
\hline
\end{tabular}


Tabla 4. Encuesta Nacional de Salud 2003. Tasas por 100 en población mayor de 17 años (resumen datos nutricionales)

\begin{tabular}{lccc}
\hline Condición & Hombres & Mujeres & Total \\
\hline Obesidad & 19,4 & 27 & 23,2 \\
Obesidad mórbida & 0,2 & 2,3 & 1,3 \\
Sobrepeso & 43,2 & 32,7 & 37,8 \\
Enflaquecidos & 0,6 & 1,1 & 0,8 \\
E. nutricional normal & 36,8 & 39,2 & 38,2 \\
Hipertensión arterial & 36,7 & 30,8 & 33,7 \\
COL total elevado & 35,1 & 35,6 & 35,4 \\
HDL disminuido & 48,4 & 30,6 & 39,3 \\
TG aumentados & 34,1 & 20,0 & 27,0 \\
Sedentarismo & 88 & 91 & 89 \\
\hline
\end{tabular}

y particulares subvencionadas. Estos datos son recopilados a partir de una encuesta que contempla la medición del estado nutricional del alumno por parte del profesor, el que está debidamente capacitado. El mapa nutricional de JUNAEB recientemente publicado revela que en primer año básico existe un $35 \%$ de niños (as) en situación de sobrepeso u obesidad. El incremento de las cifras de obesidad en este grupo aumentó de un 7 a un 16,9\% en los últimos 15 años, con sólo un 3,3\% de bajo peso.

Estudios de Atalah, Urteaga y Rebolledo en escolares de Aysén revelan que más de la mitad de la población analizada presenta algún grado de exceso de peso. Esta proporción supera lo descrito en todos los estudios anteriores, confirmando que existe una tendencia generalizada a nivel nacional a incrementar esta patología. La falta de estudios regionales previos en edades similares, impide establecer el ritmo de incremento anual de la tasa, aunque a partir de otras fuentes de información se puede estimar en 1 a 2 puntos porcentuales por año. Otro resultado relevante fue que la prevalencia de obesidad no se asoció con ninguna de las variables estudiadas: tipo de colegio, edad, curso, comuna, sexo, esto reafirma otros resultados que demostraron que la "epidemia de obesidad" es relativamente universal, en diversas condiciones socioeconómicas y ambientales. A diferencia de estudios anteriores no encontraron variaciones importantes de género, ello podría explicarse porque la mayor parte de la muestra actual fue obtenida en la etapa prepuberal, donde las diferencias tienden a ser menores. Este estudio mostró cifras de obesidad muy superiores a las observadas en la población preescolar controlada en el SNSS en el mismo período. A los cinco años de edad se encontró $23 \%$ de obesidad, superando en más de dos veces la prevalencia informada en el sistema público de salud en niños de 2 a 5 años (10\%). Debe tenerse presente que el SNSS no analiza en forma desagregada la prevalencia entre $\operatorname{los} 2 \mathrm{y}$ 6 años y la tasa promedio de este grupo pudiera no reflejar bien la prevalencia en los niños de mayor edad.

El continuo aumento en las cifras de malnutrición por exceso en la etapa escolar se ha asociado a los estilos de vida de esta población caracterizados al igual que en el adulto, por sedentarismo, con un gran número de horas frente al televisor, y hábitos alimentarios poco apropiados, con bajo consumo de verduras, frutas y lácteos y un alto consumo de alimentos procesados de alta densidad energética. La alta cobertura del sistema escolar chileno ofrece una excelente oportunidad para la educación alimentaria y nutricional. Un estudio de FAO en 50 países de América Latina, Asia y África en 1998, encontró que Chile, como la mayoría de los países no ha implementado esta enseñanza en forma sistemática debido principalmente a la falta de profesores capacitados y de materiales educativos apropiados. De este estudio nace la iniciativa entre FAO, INTA-Universidad de Chile y Mineduc de un proyecto de "Educación en Alimentación y Nutrición para la Enseñanza Básica" cuyos resultados revelaron que es posible intervenir exitosamente en esta población.

El Ministerio de Educación además está realizando una primera evaluación de diagnóstico de salud en escolares a más de 250000 escolares de primero medio los serán examinados para detectar posibles problemas cardíacos y para evitar que se produzcan muertes evitables por causa cardiovascular. Este diagnóstico nacional de salud se realizará en todos lo establecimientos municipales y subvencionados de primero medio y sus resultados estarán disponibles durante el mes de mayo del presente año.

Por otro lado, parece indispensable la capa- 
citación continua de los equipos de salud y educación para asegurar que los datos disponibles corresponden verdaderamente a la situación descrita. Un estudio de Schonhaut, Rodríguez y Pizarro en 416 alumnos de primero básico de la comuna de Colina reveló una prevalencia de sobrepeso más obesidad evaluado por el equipo de salud de un $29 \%$ vs 38\% según equipo de educación lo que constituye una diferencia significativa $(p<0,05)$. La prevalencia de bajo peso fue $4,4 \%$ según las escuelas y $5,8 \%$ según equipo de salud escolar, diferencia estadísticamente no significativa. Al comparar la concordancia para el diagnóstico nutricional entre ambos grupos según IMC/edad cronológica, se obtuvo un índice kappa de 0,56 (IC: $0,49-0,63)$, esto indica que existe discordancia en el diagnóstico en casi la mitad de los casos.

\section{Importancia de la Valoración de la Talla en el Escolar y Adolescente}

La estatura final de un sujeto viene determinada en los genes y su adecuada expresión depende de factores ambientales. El crecimiento estatural del niño es un buen indicador de la calidad del ambiente en el cual ha vivido, correlacionándose claramente con el nivel socioeconómico (NSE) y con el grado de desarrollo del país.

La talla, como indicador global de calidad de vida, se aprecia en la menor estatura de preescolares de comunas pobres al compararlos con los de comunas de ingresos altos, y en la menor estatura de adolescentes y adultos jóvenes de NSE bajo comparados con los de NSE altos. En estudios poblacionales, la mayor estatura se asocia a una mayor capacidad de trabajo físico y productividad laboral, a una menor deserción y mejor rendimiento escolar e intelectual.

El impacto que produce la desnutrición calórico-proteica postnatal temprana sobre el crecimiento de niños chilenos puede apreciarse en lo observado en un seguimiento de niños que ingresaron a CONIN para su recuperación nutricional: a los 9 años de edad presentaban una adecuación ponderal normal o excedida, pero una talla significativamente menor que la de sus pares que no tuvieron desnutrición; es decir, hubo una recuperación adecuada o excedida del peso, pero no de la estatura.
Un ambiente desfavorable dificulta la visualización de la influencia de los factores genéticos sobre el crecimiento. Sin embargo, es evidente que los factores genéticos participan sustancialmente en la determinación tanto del crecimiento estatural como del desarrollo puberal. Esto se ha observado al comparar los tiempos de maduración sexual y la magnitud del estirón puberal en diferentes grupos étnicos y en el dimorfismo sexual en la estatura de escolares chilenos, observándose un mayor retraso de talla en las mujeres que en los varones de NSE medio-bajo y bajo. Es difícil separar la influencia que ejercen los factores genéticos y los ambientales en el crecimiento de una población; sin embargo, la tendencia secular de la estatura permite visualizar la variación del componente ambiental, lo que es de importancia en los programas dirigidos a proteger a los grupos de mayor riesgo. Poblaciones que muestran una tendencia secular hacia un mayor crecimiento estatural están superando factores ambientales adversos sobre el crecimiento.

Chile muestra una clara tendencia a mejorar la estatura promedio de su población en más o menos $1,5 \mathrm{~cm}$ cada 10 años, lo que representa entre un tercio a la mitad del incremento secular de la talla de poblaciones de países desarrollados, que han llegado a un bienestar socioeconómico óptimo. Estadísticas de diferentes Organizaciones No Gubernamentales (ONG) coinciden con los antecedentes aportados por el Ministerio de Salud, en el sentido que el problema de déficit de talla en la población infantil se encuentra en descenso.

Hace 15 años, un 33\% de los escolares presentaba un retraso estatural (menos del 95\% de adecuación de la talla para la edad, según tablas de la OMS) lo que ha ido descendiendo a alrededor del 23\%. Esta mejoría ha coincidido con un franca mejoría de la salud de estos niños en Chile, presentando una notoria disminución de la mortalidad infantil, de las tasas de desnutrición, y de las enfermedades infecciosas. Actualmente el ambiente es más propicio para que los niños expresen su potencial genético de crecimiento, lo que ha permitido detectar la existencia de un dimorfismo sexual en el retraso estatural de nuestra población infantil, siendo más marcado el retraso estatural en las muje- 
res que en los varones, especialmente en los estratos socioeconómicos bajos, lo que implicaría una influencia étnica en la estatura de nuestros escolares.

En preescolares que asisten a jardines pertenecientes a la JUNJI el retraso de talla ha disminuido entre los años 1986 y 1994 de 37\% a 17\%. En escolares de educación básica apoyados por los programas de la JUNAEB, el retraso estatural ha disminuido de 33 a $20 \%$ entre los años 1989 y 1994. Si bien los grupos protegidos por programas de apoyo nutricional muestran una clara tendencia a la disminución del retraso de talla, varios estudios nacionales muestran déficit nutricionales específicos especialmente en grupos etáreos no protegidos como los adolescentes.

Estudios sugieren el rol de un factor genético en la pubertad más temprana y en el menor estirón puberal de escolares chilenos, al compararlos con americanos y europeos, que se traduciría en una menor talla final. Estudios de Burrows, Ramírez y Cordero muestran un cambio secular hacia una mayor estatura en los escolares de la II, IX y XIII Regiones del país. Esto podría estar traduciendo una mejoría en la calidad del ambiente en que ellos han vivido estos últimos años. Un fenómeno similar fue observado en los japoneses y mexicanos que inmigraron a EE.UU., donde la población que se crió en este país alcanzó estaturas mayores que las de su ascendencia que vivió su niñez en su país de origen, donde las condiciones ambientales eran adversas.

Se ha señalado que los mayores cambios en la estatura del ser humano se han producido durante este último siglo, por lo que se aconseja hablar de "cambio secular" y no de tendencia, ya que esta última se refiere a cambios mantenidos y constantes a través del tiempo. Estudios realizados sobre la variación del crecimiento humano durante este siglo, en países desarrollados y en vías de desarrollo, señalan que este "cambio" hacia una mayor estatura se asocia a una mejor nutrición, atención sanitaria, control de infecciones, reducción del número de hijos y movilidad poblacional, y que los países desarrollados están estabilizando su crecimiento.

En el referido estudio de Burrows y cols, se observa que el crecimiento aún no se ha estabi- lizado, ya que entre los años 1991 y 1994 se observa una disminución todavía significativa del retraso estatural y los escolares mayores son el grupo con mayor retraso. Esta situación es propia de países que aún no han alcanzado una calidad de vida óptima. Si bien, el estudio del cambio secular del estado nutricional muestra un claro aumento en la prevalencia de obesidad en los escolares chilenos, es necesario evaluar otros factores ambientales asociados al crecimiento, como el déficit de nutrientes específicos y la escasa actividad física, que son situaciones ya descritas en escolares chilenos y que pueden estar conviviendo con la obesidad. Mejorar la calidad del ambiente es la mejor estrategia para seguir mejorando la expresión del potencial genético de crecimiento del niño chileno.

\section{Alimentación}

En Chile, no contamos con una encuesta nacional de consumo, existen estudios en diversos grupos que revelan que los hábitos alimentarios de nuestra población son poco saludables. Atalah en la región de Aysén reveló en 340 niños, de kinder a $4^{\text {to }}$ año básico, un bajo consumo de verduras, frutas y lácteos y un alto consumo de azúcares. Orellana por otro lado, en un estudio de ingesta en preescolares que asisten o no al jardín infantil demostró una ingesta energética superior a las necesidades (mayor en el grupo que asiste), con un alto consumo de golosinas en ambos grupos. Yáñez, Olivares y cols, en un estudio en niños de segundo ciclo de enseñanza básica, demostraron consumo excesivo de cereales, aceites y azúcares e insuficiente de lácteos, verduras y frutas, en relación a las recomendaciones de la pirámide alimentaria. Esta información es concordante en todos los estudios disponibles y en las distintas edades, con algunas diferencias geográficas a lo largo del país, según acceso a los distintos alimentos.

\section{Actividad Física}

El programa Vida Chile uno de cuyos principales objetivos es la promoción de la salud, estableció para el año 2010 es la disminución de la obesidad en escolares de primer año básico de $17 \%$ en el año 2000 a $12 \%$ en el 2010 
También se estableció una meta relacionada con actividad física con una disminución importante del porcentaje de población sedentaria.

Sin duda que los programas a nivel escolar, diseñados e implementados correctamente, pueden ejercer un rol muy importante en la promoción de la salud En ese sentido, la escuela promotora de salud representa una estrategia que pretende involucrar a toda la comunidad educativa, a través de la realización de actividades regulares en tres condicionantes en forma simultánea con al menos 50\% del alumnado. Al año 2002, alrededor de 620000 escolares de todo el país se beneficiaron de esta estrategia.

En ese contexto, el año 2002, el INTA de la Universidad de Chile, con el apoyo técnico y financiero del Ministerio de Educación (MINEDUC), el Instituto Nacional del Deporte (Chiledeportes), el Ministerio de Salud (MINSAL), realizó un estudio diagnóstico de la situación nutricional, de actividad física y de educación ambiental en escolares de educación básica municipalizados, como asimismo determinó la factibilidad de que las escuelas seleccionadas sean "Escuelas Libres de Humo de Tabaco". Posteriormente se desarrolló, implementó y evaluó un proyecto piloto en promoción de la salud durante el año 2002, dirigido a escolares de educación básica municipalizada que incluyó cuatro componentes: alimentación, actividad física, tabaquismo y medio ambiente.

Los resultados referentes al estado nutricional de estos escolares fueron similares a otros previos, provenientes de una muestra de escolares también de escuelas municipales de 3 ciudades del país. En esa muestra (incluyó niños de $3^{\circ}$ a $7^{\circ}$ básico), la prevalencia de obesidad en los grupos de $8-9,10-11$ y 12-13 años fue la siguiente: $21,3 \%, 16,2 \%$ y $13,5 \%$ y $15,4 \%, 12,9$ y 11,7 en hombres y mujeres respectivamente. Bellizzi y cols, que analizaron datos antropométricos en escolares entre 10 y 18 años, de varios países asiáticos y europeos, observaron que la prevalencia de obesidad era mayor en los hombres hasta los 15 años, situación que se revertía en los mayores de esa edad. Los resultados del estudio del INTA mostraron que a partir de los 12 años, la prevalencia de obesidad es igual en ambos sexos.

Se ha demostrado que el nivel socioeco- nómico es un factor determinante de la actividad física, y que podría existir una asociación entre ésta y lo que generalmente ocurre con escolares de escuelas públicas, quienes tienen menos horas de clases de educación física o menos actividad física fuera del colegio. Aranceta y cols, mostraron que escolares españoles con madres de bajo nivel educacional desarrollaban menor actividad física y miraban más televisión. En nuestro país, Godoy analizó los resultados de estudios sobre capacidad aeróbica en escolares de diferente nivel socioeconómico publicados previos a 1999, concluyendo que existía una asociación directa entre ambas variables. Es decir, la potencia aeróbica aumentaba linealmente con la edad y era mayor en escolares provenientes de colegios particulares. Sin embargo, cuando este mismo investigador realizó un estudio en 2001, que incluyó a 273000 estudiantes de 6 a 19 años de escuelas municipalizadas, subvencionadas $\mathrm{y}$ particulares de todo el país, mostró que aún cuando se observaba una tendencia a mejores resultados en los colegios particulares, las diferencias no fueron significativas por edad y sexo.

Los resultados obtenidos en el estudio INTA muestran la alta prevalencia de obesidad en escolares de educación básica, especialmente en los menores y lo alejada que están estas cifras de la meta propuesta por Vida Chile. Además, se demuestra el deficiente nivel de aptitud física. Estos hallazgos son muy preocupantes, ya que la mayoría de los hábitos de vida se forman en la niñez e indican la imperiosa necesidad de realizar acciones que impacten estos aspectos.

\section{Trastornos de la conducta alimentaria}

En Chile, no disponemos de información nacional sobre la prevalencia de estos trastornos. Estudios de Behar, aplicando Tests de Actitudes Alimentarias y de Trastornos Alimentarios, que evalúan el riesgo de evolucionar hacia algún trastorno de la conducta alimentaria, encontró en escolares de III y IV Medio de la V región del país que el riesgo era de un $18 \%$, en universitarias era $15 \%$ y en adolescentes con sobrepeso era del $41 \%$.

A la edad escolar aparece la preocupación 
por la imagen corporal, la que se asocia con popularidad, inteligencia y éxito y se rechaza la gordura. Esta situación se observa con mayor frecuencia en las mujeres, las que centran su ideal en un cuerpo delgado, en la prevalente focalización en la apariencia externa y la trascendencia de esta figura en el éxito social, tendencia que se acentúa durante la adolescencia. Por otro lado, los hombres también muestran aversión por el sobrepeso, pero con menos preocupación por engordar y no expresan tanta insatisfacción con algunas partes de su cuerpo como lo hacen las mujeres.

Se estima que la incidencia de la anorexia nervosa ha aumentado en los últimos decenios, en especial en los países occidentales industrializados. También se ha comprobado que es mayor en el nivel socioeconómico medio y alto. Un $85 \%$ de los pacientes desarrollan la anorexia entre los 13 y los 20 años de edad. La epidemiología de la bulimia es más compleja, en parte porque su delimitación como entidad nosológica es más reciente, por lo que los estudios de prevalencia difieren por usar distintos criterios diagnósticos y también porque muchos casos pueden mantenerse ocultos, al no haber compromiso del peso corporal. Su edad de inicio es generalmente más tardía, siendo más frecuente en los últimos cursos del colegio y primeros de la universidad (18 años). Variantes leves en adolescentes (variantes y problemas del DSM-PC) ocurren en un 5 a $10 \%$ adicional.

Estudios de Behar en deportistas revelan que el $18,1 \%$ de los sujetos deportistas, la mayoría de ellos de sexo femenino $(93,1 \%$ versus $6,9 \%$ de sexo masculino) obtuvo puntajes en rango patológico en tests de TCA, porcentaje bastante más elevado de lo esperable en una población general en la que oscilan entre $0,5 \%$ y $1 \%$ para la anorexia nerviosa y entre $1 \%$ y $3 \%$ para la bulimia nerviosa; pudiendo presumirse con esto que este grupo sería portador de una patología alimentaria presente en la actualidad o un grupo de riesgo de un desorden alimentario en el futuro bajo ciertas contingencias favorecedoras. La implicancia más relevante de este estudio es el análisis de que una población que practica una actividad física regular, lo cual podría considerarse como una costumbre saludable en el contexto de un estilo de vida, podría transformarse en un peligro de padecer trastornos de la conducta alimentaria.

Este y otros estudios enfatizan la necesidad de utilizar programas no sólo de promoción de salud sino también estrategias de detección precoz y prevención primaria psicoeducativa en estos grupos riesgosos, particularmente en preadolescentes y adolescentes, incluyendo a los padres, profesores, entrenadores y equipos de salud. El ejercicio extremo parece ser un factor de riesgo para desarrollar una anorexia nerviosa, especialmente cuando se combina con la realización de una dieta. Se ha identificado una "tríada atlética femenina" que consiste en estado de agotamiento, depresión e irritabilidad, con la cual los atletas continúan entrenando pero su rendimiento disminuye. Ambos han sido relacionados al síndrome de "anorexia de la actividad" que ha sido observada en modelos animales. Para estos grupos de riesgo, algunas preguntas simples de sondeo acerca del peso, posible insatisfacción con la apariencia, amenorrea y la ingesta nutricional del día antes de la evaluación, pueden ayudar a identificar el desarrollo de una patología alimentaria. La intervención precoz tanto médica como psiquiátrica son claves para la pronta recuperación.

\section{Enfoque sicosocial del escolar y adolescente}

Por último, cuando nos referimos a escolares y adolescentes no podemos ignorar que hay distintos factores sicosociales que los afectan en todos los ámbitos del quehacer, incluido el ámbito de la salud.

Una revisión del tema de Jadue revela que factores como las transformaciones familiares, incrementando en los hijos el riesgo de bajo rendimiento, de fracaso, de problemas emocionales y conductuales en la escuela, señalan la apremiante necesidad de que en el sistema escolar se desarrollen mecanismos protectores para los niños provenientes de familias monoparentales y disfuncionales, a través de la implementación de redes de apoyo, constituidas por talleres de desarrollo personal, por el ejercicio de un estilo democrático de docencia y la comunicación eficiente entre las familias y la escuela.

Dentro de un mundo globalizado, en Chile, al 
igual que muchos otros países, se desarrolla el proceso de modernización. Modernización se refiere a los avances tecnológicos de racionalización y a la transformación del trabajo y de la organización e incluye muchos otros cambios: el cambio de los caracteres sociales y de las biografías, de los estilos de vida, de las formas de amar, de las estructuras de influencia y de poder, de las formas políticas de opresión y de participación, de las concepciones de la realidad y de las normas cognoscitivas. Todos estos cambios transforman las fuentes de la certeza de que se nutre la vida. En este contexto, la valoración que el niño (a) hace de sí mismo (autoestima) y las cogniciones acerca de sí mismo (autoconcepto) están en la base del desarrollo de la competencia emocional.

Sin duda que estos aspectos un poco alejados de nuestra tarea diaria afectan más primordialmente la salud de este grupo etario enfrentado a los cambios que trae consigo la pubertad y la adolescencia y los hace más vulnerables a TCA como los descritos transformándolos en grupos de riesgo que debemos vigilar y proteger.

\section{Comentario Final}

Entre las conclusiones de esta revisión cabe destacar que para los escolares y adolescentes contamos con pocos datos estadísticos y menos aún de intervención, ya que son grupos que tradicionalmente han estado más alejados de los centros primarios de atención de salud.

Por otro lado, el desarrollo de cualquier estrategia de evaluación e intervención a esta edad necesariamente obliga a coordinarse efectivamente con el ámbito de la educación, quienes son los principales protagonistas en la vida de estos niños (as).

Se ha avanzado en la forma de diagnóstico, el paso siguiente es desarrollar estrategias de capacitación a nivel escolar para que esta evaluación se haga efectiva y eficiente, y el paso siguiente es planificar en conjunto, salud y educación, las actividades para ambos ámbitos, en relación a promoción de salud, detección de factores de riesgo y prevención de los trastornos que afectan a estos grupos.
La "Escuela Saludable" representa una excelente oportunidad de promover cambios en los hábitos alimentarios, del niño y la familia, a través del sistema escolar. Ello necesariamente debe complementarse con otras acciones tanto de salud, de educación y de otras áreas la sociedad, dirigidas a la toda la comunidad, considerando que al ingresar a la escuela cerca de la mitad de los niños ya presenta sobrepeso.

La participación comunitaria, el autocuidado de la salud y las consideraciones del área de la salud mental deben ser elementos fundamentales en cualquier intervención que espere obtener éxito.

\section{Referencias}

1.- Burrows AR, Díaz SN, Muzzo S: Variaciones del índice de masa corporal (IMC) de acuerdo al grado de desarrollo puberal alcanzado. Rev Méd Chile 2004; 132 : 1363-8.

2.- Norma Técnica de Evaluación Nutricional de Niños y Niñas de 6 a 18 Años. Ministerio de Salud Chile 2004 (www.minsal.cl).

3.- Encuesta Nacional de Salud. Ministerio de Salud Chile 2003 (www.minsal.cl).

4.- Mapa Nutricional de Junaeb, Escolares de Primero Básico, Establecimientos Municipalizados y Particulares Subvencionados, según región, comuna y escuela 2002. www.junaeb.cl.

5.- Educación en Alimentación y Nutrición para la Enseñanza Básica. FAO, Ministerio de Educación Chile, INTA-U. de Chile, 2003.

6.- Aburto AM: Desafíos del Programa de Alimentación Escolar. Conferencia del tercer curso de Nutrición del escolar y adolescente, Sociedad Chilena de Pediatría, abril 2001: 93-100.

7.- Hodgson MI: Obesidad en el escolar y adolescente. Conferencia del tercer curso de Nutrición del escolar y adolescente, Sociedad Chilena de Pediatría, abril 2001: 63-70.

8.- Vio del RF, Albala C: Epidemiología de la Obesidad en Chile. Rev Chil Nutr 2000; 27 (51): 97-104.

9.- Schonhaut L, Rodríguez L, Pizarro T: Concordancia en el diagnóstico nutricional según índice de masa corporal, entre el equipo de salud y las escuelas de la Comuna de Colina. Rev Chil Pediatr 2004; 75: 32-5.

10.- Burrows R, Leiva L, Giadrosic V, et al: Estado nutricional y adecuación estatural de escolares en etapa puberal de diferentes regiones del país. Rev Chil Nutr 1992; 20: 212-8.

11.- Burrows R, Muzzo S: Curvas de crecimiento del escolar chileno de 5 a 16 años por NSE. Rev Chil Nutr 1996; 24: 1 .

12.- Burrows A, Ramírez MR, et al: Cambio secular del 
retraso de talla en escolares chilenos de tres regiones del país. Rev Chil Pediatr 1999; 70: 390-7.

13.- Marín V: Trastornos de la conducta alimentaria en escolares y adolescentes. Rev Chil Nutr 2002; 29: 8691.

14.- Behar AR, Hernández TP: Deportes y trastornos de la conducta alimentaria. Rev Méd Chile 2002; 130: 287 94.

15.- Behar AR: Trastornos del hábito de comer en adolescentes: aspectos clínicos y epidemiológicos. Rev Méd Chile 1998; 126: 1085-92.

16.- Behar AR, De la Barrera CM, Michelotti CJ: Identidad de género y trastornos de la conducta alimentaria. Rev Méd Chile 2000; 129: 1003-11.

17.- Jadue JG: Transformaciones familiares en Chile: riesgo creciente para el desarrollo emocional, psicosocial y la educacion de los hijos. Estud Pedagóg 2003; 29: 115-26.

18.- Muzzo BS: Crecimiento normal y patológico del niño y del adolescente. Rev Chil Nutr 2003; 30: 92-100.

19.- Kain J, Olivares $S$, Romo M, et al: Estado Nutricional y resitencia aeróbica en escolares de educación básica: línes d ebase de un proyecto de promoción de la salud. Rev Méd Chile 2004; 132: 1395-402.

20.- Parodi ML, López ME, Capurro MT: Prevalencia de obesidad en escolares de enseñanza básica. Rev Chil Pediatr 1993; 64: 79-183.

21.- Díaz E, Burrows R, Muzzo S, Galgani J, Rodríguez R: Evaluación nutricional de adolescentes mediante índice de masa corporal para etapa puberal. Rev Chil Pediatr 1996; 67: 153-8.

22.- Burrows R, Giadrosic V, Biolley E, et al: Estado nutricional y adecuación estatural de escolares en etapa puberal de diferentes regiones de Chile. Rev Chil Nutr 1992; 20: 212-8.

23.- Ivanovic R, Olivares $M$, Ivanovic D: Estado nutricional en escolares chilenos urbanos y rurales de la Región Metropolitana, 1986-1987. Rev Chil Pediatr 1990; 61: 210-7.

24.- Atalah SE, Urteaga $R$, et al: Prevalencia de obesidad en escolares de la Región de Aysén. Rev Chil Pediatr 1999; 70: 208-14. 